\title{
Quantification of light Polycyclic Aromatic Hydrocarbons in seafood samples using on-line Dynamic HeadSpace extraction, ThermoDesorption, Gas Chromatography tandem Mass Spectrometry, based on an isotope dilution approach
}

\author{
Guiffard Ingrid 1, ${ }^{*}$, Geny Thomas ${ }^{1}$, Veyrand Bruno ${ }^{1}$, Marchand Philippe 1, Pellouin-Grouhel Anne 2, \\ Bizec Bruno Le ${ }^{1}$, Bichon Emmanuelle ${ }^{1}$
}

${ }^{1}$ LABERCA, Oniris, INRA, Route de Gachet, CS50707, 44307 NANTES cedex 3, FRANCE

2 IFREMER, Laboratory of Biogeochemistry and ecotoxicology, Rue de I'lle d'Yeu, BP 21105, 44311

NANTES cedex 3, FRANCE

* Corresponding author : Ingrid Guiffard, email addresses : ingrid.guiffard@oniris-nantes.fr ; laberca@oniris-nantes.fr

\begin{abstract}
:
The aim of our work was to develop an analytical strategy to quantify naphthalene, acenaphthylene, acenaphthene, fluorene, phenanthrene and anthracene in fish products by on-line dynamic headspace extraction, followed by thermodesorption injection and gas chromatography analysis coupled with tandem mass spectrometry using electron ionization mode (DHS-TD-GC-EI-MS/MS). The developed protocol used $1 \mathrm{~g}$ of freeze-dried or oil sample supplemented with perdeuterated light PAHs. The sample was heated at $\left[90-100^{\circ} \mathrm{C}\right]$, the headspace of the sample was swept by nitrogen and the trapping of the PAHs was carried out on a Tenax-type adsorbent placed at $25^{\circ} \mathrm{C}$. Analytes were thermodesorbed at $300^{\circ} \mathrm{C}$ from the dried adsorbant and then cryofocused on a cooled injection system (CIS) at $\neg 25^{\circ} \mathrm{C}$ before injection $\left(12^{\circ} \mathrm{C} \mathrm{s}-1\right.$ up to $\left.300^{\circ} \mathrm{C}\right)$. The chromatographic separation of PAHs was carried out on a 5-MS type column $(30 \mathrm{~m} \times 0.25 \mathrm{~mm}, 0.25 \mu \mathrm{m})$ and the acquisition of the signals was performed in SRM following the transitions, involving the loss of one or two hydrogen atoms from the molecular ion. In view of the principle of extraction, the calibration curve was performed on a representative matrix or using the standard addition method. Quantification limits were determined between 0.01 and $0.6 \mathrm{ng} \mathrm{g}-1$ of matrix from the method blank results. The method was validated by a series of multi-level supplemented matrix assays and by the analysis of a reference material from an inter-laboratory test (mussels, IAEA-432). The average of the expanded measurement uncertainty was from 9 to $44 \%$ for the four lightest PAHs, except for fluorene when the sample incubation was set at $90^{\circ} \mathrm{C}$. Occurrence measurements were performed on almost two hundred samples of molluscs, echinoderms and fish. The results have shown a quantification frequency greater than $66 \%$ for naphthalene and fluorene, at concentrations below a few ng g-1 of dry matter of fishery products. With this methodology, the light PAHs occurrence can now be measured in a wider range of foodstuffs in order to better characterize their contamination trends and the associated risk simultaneously.
\end{abstract}




\section{Highlights}

- A DHS-TDU-GC-MS/MS method was developed to quantify light PAHs in seafood $\boldsymbol{}$ Two methods are now available, one is more accurate and the second more robust Validation was performed to determine measurement uncertainties at [1-20] $\mathrm{ng} \mathrm{g}^{-1} \mathrm{dw} \triangleright$ A first occurrence level was measured in around 200 seafood samples

Keywords : Light Polycyclic Aromatic Hydrocarbons (PAHs), Dynamic HeadSpace (DHS), Thermo Desorption (TDU), GC-MS/MS, isotope dilution, seafood 


\section{Introduction}

Polycyclic Aromatic Hydrocarbons (PAHs) are organic compounds constituted of at least two condensed aromatic hydrocarbonated rings. Their natural and anthropic sources, resulting from pyrogenic and petrogenic inputs, lead to their global release into the environment [1-3]. Food processing can also induce PAHs contamination, as in grilled food or in smoked fish [4]. Moreover, food from animal origin can be contaminated by bioaccumulation of PAHs all along the food chain, because of their lipophilic properties [5]. PAHs human exposure is of major concern since their toxicity was demonstrated in regard to their carcinogenicity, teratogenicity and mutagenicity [6].

The US Environmental Protection Agency first, and then the European Food Safety Agency, have determined priority lists which are more focused on the heaviest PAHs [7], because they represent greater harmful effects [8]. Therefore, the PAHs exposure assessment studies found in literature more often reported occurrence of « heavy PAHs » with at least four benzene rings [9]. However, light PAHs, made up of 2 or 3 condensed rings (cf. Fig. 1), are also of scientific concern. Actually, naphthalene (NAP) is classified 2B whereas acenaphtene (ACE), fluorene (FLU), phenanthrene (PHE) and anthracene (ANT) are classified 3 in the International Agency for Research on Cancer (IARC) classification [10]. Their occurrence in the total environment was already reported thanks to the US EPA list which includes these compounds. Obviously, their human exposure is more correlated to air contamination because of their volatility, particularly naphthalene, fluorene and phenanthrene [11]. Nevertheless, food remains a significant way of Human exposure. A Spain dietary exposure study conducted in 2006 has reported the prevalence of light PAHs compared to heavy PAHs in food [12]. Human biomonitoring studies have also confirmed the relevance to monitor these light PAHs. Actually, Thai et al. [13] found significant amount above the tens of $\mu \mathrm{g} \mathrm{L}^{-1}$ of $\mathrm{OH}$-naphtalene in urine in the Australian population. This biomarker was present at concentrations ten times higher than those observed for the other metabolites of light PAHs.

Multiresidue methods have been developed in the last two decades to determine and monitor PAHs exposure according to the regulation, mainly using GC-MS/MS analysis [14, 15] to discriminate isomers. In most cases, the sample preparation included Pressurized Liquid 
Extraction (PLE) [16] or Soxhlet extraction [17] to maximize recovery yields before purification. However, these extraction steps are critical for light PAHs. On the one hand, losses of light PAHs were demonstrated during solvent evaporation. On the other hand, naphthalene contamination remains an important issue in the laboratory because of its ubiquitous behaviour. PLE instruments but also evaporators and indoor air could contribute to increase the environmental contamination of naphthalene. Many different sample preparation methods have been proposed in the literature [18], among which SPME has been suggested as an interesting alternative to monitor light PAHs . They described mixtures of DVB, Carbon and PDMS adsorbents to achieve PAHs extraction from vegetable oil [19, 22], milk [20], fish [21] or smoked food [23]. The sample preparation was carried out in a closed headspace vial where the external contamination was kept under control. Moreover, no additional evaporation step was required and the light PAHs were preserved on the SPME fiber before analysis. However, the enrichment capability of SPME is limited to its stationary phase quantity and to the partition coefficient of PAHs between the gaseous and the stationary phases during the extraction step. SPME fibers are also known as brittle and could present carry-over issues. More recently, headspace mode of liquid phase microextraction (HS-SDME) was described as easy to implement with automation ability for PAHs extraction from water and environmental samples $[24,25]$. However, the partition coefficient between liquid and gaseous phase could also limit the enrichment capability and, thus, the sensitivity of the method.

Therefore, to shift the balance of PAH partitioning from the sample to the gaseous phase, Dynamic HeadSpace (DHS) extraction can be performed by continuously sweeping the headspace of the sample with a significant quantity of gas. Then, the extracting gas is loaded on a selective adsorbent where analytes are trapped. Thermodesorption of analytes from the trap is then required before cryofocalisation and GC-MS analysis. This approach has already been used for volatile compounds determination in ham [26] based on the work of Barcarolo and Casson [27], and also for wine studies [28, 29], for olive oil characterisation [30] or for biomonitoring studies such as chlorinated contaminants determination in human urine [31]. As a result, sensitivity was significantly improved in comparison to static headspace techniques. Moreover, the authors have validated their DHS-TDU-GC/MS methods and have demonstrated a promising intermediate precision for this kind of non-targeted approach [29, 30].

The aim of our study was to assess the DHS-TD-GC/MS/MS approach for light PAHs quantification in seafood which is quite challenging because of the complexity and diversity of such matrix. The final goal of our work is to cover the whole PAHs list, monitoring the four lightest PAHs with this new approach and the other ones with the conventional method published by Veyrand et al. in 2007 [14] in parallel. 


\section{Experimental}

\subsection{Reagents and material}

Toluene of picograde quality was obtained from LGC (Wesel, Germany). The native compounds (Naphthalene ; Acenaphthylene (ACY) ; Acenaphthene ; Fluorene ; Phenanthrene ; Anthracene ; Fluoranthene; Pyrene; Benz[a]anthracene; Chrysene ; Benzo[b]fluoranthene ; Benzo[k]fluoranthene ; Benzo[a]pyrene ; Indeno[1,2,3-cd]pyrene ; Benzo[ghi]perylene ; Dibenz[a,h]anthracene) and the perdeuterated internal standard compounds (Naphthalene- $\mathrm{d}_{8}$; Acenaphthylene- $\mathrm{d}_{8} ;$ Acenaphthene- $\mathrm{d}_{10} ;$ Fluorene- $\mathrm{d}_{10} ;$ Phenanthrene- $\mathrm{d}_{10} ;$ Anthracene- $\mathrm{d}_{10}$; Fluoranthene- $\mathrm{d}_{10}$; Pyrene- $\mathrm{d}_{10}$; Benz[a]anthracene- $\mathrm{d}_{12}$; Chrysene- $\mathrm{d}_{12}$; Benzo[b]fluoranthene$\mathrm{d}_{12} ; \quad$ Benzo[k]fluoranthene- $\mathrm{d}_{12} ; \quad$ Benzo[a]pyrene- $\mathrm{d}_{12} ; \quad$ Indeno[1,2,3-cd]pyrene- $\mathrm{d}_{12}$; Benzo[ghi]perylene- $d_{12}$; Dibenz[a,h]anthracene- $d_{14}$ ) were purchased from Dr Ehrenstorfer (Augsburg, Germany). The concentration of these commercial mixtures was $100 \mathrm{ng} \mu \mathrm{L}^{-1}$ in toluene. Successive dilutions by ten or twenty were prepared in toluene at 10,1 and $0.1 \mathrm{ng} \mu \mathrm{L}^{-1}$

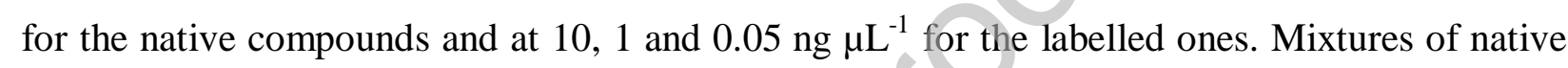

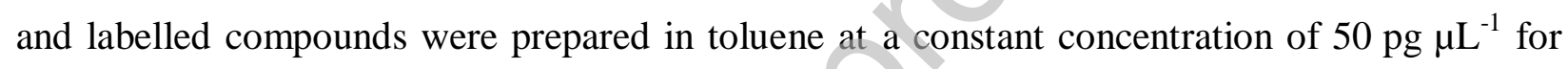

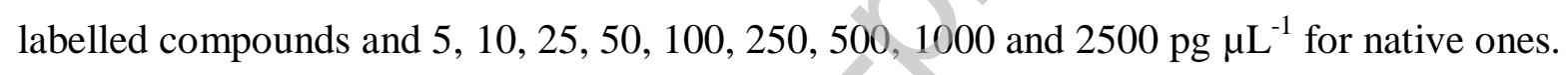

For Dynamic HeadSpace extraction, $20 \mathrm{~mL}$ headspace vials with screw caps were purchased from Gerstel (Mülheim, Germany).

Samples of mollusc, echinoderm, algae oil and fish were collected in 2017 and 2018 by the French chemical monitoring network $(\mathrm{ROCCH})$ managed by the French Marine Science Research Institute along the French coasts, and by the departmental civilian population protection services as part of the French control plan.

\subsection{Sample pretreatment}

To avoid the risk of contamination, samples were prepared in an air-conditioned room away from solvent vapor. After freeze-drying, each sample was weighed in order to determine its water content. One gram of the ground dried sample was introduced in a $20 \mathrm{~mL}$ headspace vial and spiked with $20 \mu \mathrm{L}$ of a labelled internal standard solution at $50 \mathrm{pg} \mu \mathrm{L}^{-1}$. A few samples of algae oil (expanded scope of seafood) were analyzed using the same principle associated to standard addition method. To this end, four vials were prepared with $1 \mathrm{~g}$ of the same oil sample. Twenty microliters of the labelled internal standard solution at $50 \mathrm{pg} \mathrm{L}^{-1}$ were added into the first vial, whereas the three other ones received $20 \mu \mathrm{L}$ of solutions at 50,250 or $1000 \mathrm{pg}^{-1} \mathrm{~L}^{-1}$ of

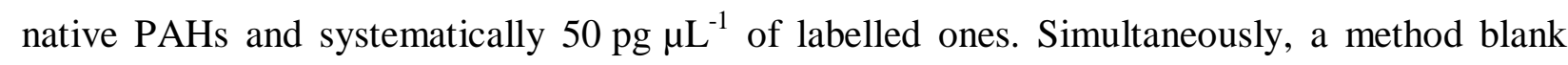
was prepared introducing only $20 \mu \mathrm{L}$ of a labelled internal standard solution at $50 \mathrm{pg} \mu \mathrm{L}^{-1}$, at the bottom of the headspace vial. For all the dried matrices, $20 \mu \mathrm{L}$ of a labelled internal standard

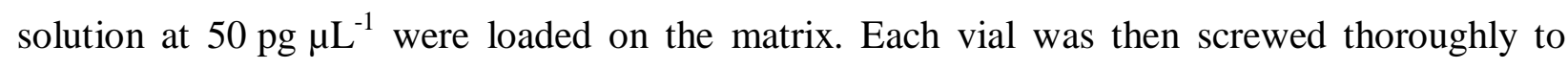


avoid any losses of the headspace phase during the extraction step. Before placing the vials into the autosampler, they were gently shaken manually without reversing the vial, in order to enhance the spiking solution impregnation and the homogeneity of the sample.

\subsection{On-line Dynamic Headspace extraction}

Firstly, to prevent any issue on the automatic dynamic headspace instrument, several precautions were implemented in our lab. Each headspace septum was pierced only once. The dry purge septum cap was changed each sequence or everyday if the sequence was longer, to keep a good tightness and to keep the required flow rate stable.

Then, two DHS methods were developed in this work, called Method 1 and Method 2.

Method 1: The vial was incubated during 10 minutes at $100^{\circ} \mathrm{C}$ under stirring $(500 \mathrm{rpm})$ before extraction. The sample was then maintained at $100^{\circ} \mathrm{C}$ during extraction without stirring. To achieve analyte extraction, $500 \mathrm{~mL}$ of nitrogen were used as sweep gas at $75 \mathrm{~mL} \mathrm{~min}^{-1}$. The Tenax TA trap and the DHS transfer line were set at $25^{\circ} \mathrm{C}$ and $150^{\circ} \mathrm{C}$ during the extraction step respectively. The Tenax TA trap was then slightly heated at $50^{\circ} \mathrm{C}$ and dried with $1 \mathrm{~L}$ of nitrogen

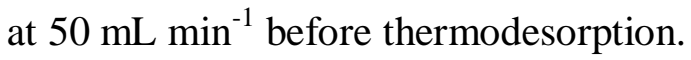

Method 2: The vial was incubated during 10 minutes at $90^{\circ} \mathrm{C}$ under stirring $(500 \mathrm{rpm})$ before extraction. The sample was then maintained at $90^{\circ} \mathrm{C}$ during extraction without stirring. To achieve analyte extraction, $2 \mathrm{~L}$ of nitrogen were used as sweep gas at $100 \mathrm{~mL} \mathrm{~min}^{-1}$. The Tenax TA trap and the DHS transfer line were set at $25^{\circ} \mathrm{C}$ and $150^{\circ} \mathrm{C}$ during the extraction step respectively. The Tenax TA trap was then slightly heated at $50^{\circ} \mathrm{C}$ and dried with $2 \mathrm{~L}$ of nitrogen at $100 \mathrm{~mL} \mathrm{~min}^{-1}$ before thermodesorption.

\subsection{Thermodesorption injection}

The trap was thermodesorbed in the TDU programmed from $30^{\circ} \mathrm{C}(0.1 \mathrm{~min})$ up to $300^{\circ} \mathrm{C}(5 \mathrm{~min})$ at $60^{\circ} \mathrm{C} \mathrm{min}{ }^{-1}$. The TDU transfer line was set at $300^{\circ} \mathrm{C}$ and analytes were refocused in the CIS equipped with a baffle liner and cooled at $\neg 25^{\circ} \mathrm{C}$ by a chiller. GC injection was performed thanks to a fast heating of the CIS up to $300^{\circ} \mathrm{C}(5 \mathrm{~min})$ at $12^{\circ} \mathrm{C} \mathrm{s}^{-1}$.

\subsection{DHS-TDU-GC-MS/MS}

A robotic arm MultiPurpose Sampler (MPS) was equipped with a DHS system [32] and a ThermoDesorption Unit (TDU) coupled to a Cooled Injection System (CIS), all from Gerstel company (Mülheim, Germany) [33]. The GC-MS/MS instrument consisted in a gas chromatograph Agilent 7890 Series coupled to a triple quadrupole analyzer Agilent 7000 operating in electron ionization $(70 \mathrm{eV})$. Gas chromatography was performed on a non-polar column DB-5MS $(30 \mathrm{~m} \times 0.25 \mathrm{~mm}, 0.25 \mu \mathrm{m})$ purchased from Agilent (Santa Clara, CA USA). Helium was used as carrier gas at a constant flow rate of $1 \mathrm{ml} \mathrm{min}^{-1}$. The gradient of temperature was $90^{\circ} \mathrm{C}(2 \mathrm{~min}), 10^{\circ} \mathrm{C} \mathrm{min}^{-1}$ to $240^{\circ} \mathrm{C}(0 \mathrm{~min})$ and $40^{\circ} \mathrm{C} \min ^{-1}$ to $320^{\circ} \mathrm{C}(10 \mathrm{~min})$. The $\mathrm{GC}$ 
transfer line was maintained at $300^{\circ} \mathrm{C}$. Temperature of the source was kept at $230^{\circ} \mathrm{C}$. A mix of Nitrogen and Helium was used as collision gas. Two transitions per PAH were selected (cf. Table 1 and Fig.S1).

\subsection{Identification}

The following requirements have to be fulfilled before light PAHs quantification [34]: presence of internal standard $(\mathrm{S} / \mathrm{N}>3)$, acceptable relative retention time of the analyte (tolerance of $\pm 0.5 \%$ ), detection of the two diagnostic transitions ( $\mathrm{S} / \mathrm{N}>3$ ), acceptable intensity ratio of the two diagnostic transitions (tolerance from 20 to $50 \%$ depending on the ratio).

\subsection{Quantification}

The quantification was based on the isotope dilution approach. Each native compound amount was determined taking into account each corresponding labelled compound as internal standard. The calibration was performed using a similar matrix spiked at 10 different levels for native compoundsincluding 0 and constant level for labelled ones (cf. Fig. S2a), as described in the "reagents and material" part. The calibration used an unweighted method and each calibration point was injected once, at the beginning of each sequence. As calibration curves were built with a pool of similar matrix, only slopes were used to determine concentrations. Using the standard addition method, the calibration curves being built with the sample matrix, amounts were calculated dividing intercepts by slopes (cf. Fig. S2b). Method blanks were quantified with a calibration without matrix.

\subsection{Validation procedure}

Each validation was based on three series of four-level supplemented matrix assays (pool of dried mussels) at $0,1,5$ and $20 \mathrm{ng}$ for native compounds, resulting in $\mathrm{n}=6,10,6$ and 6 samples respectively. A reference material coming from an inter-laboratory test (mussels, IAEA-432) was included once in each series. Each performance criterion was assessed at each level and averaged. We classically used the following definitions: the limit of detection (LOD) corresponds to amounts for which the first transition presents a signal-to-noise ratio $(\mathrm{S} / \mathrm{N})$ of 3 , and limit of quantification (LOQ) corresponds to amounts for which the second transition presents a $\mathrm{S} / \mathrm{N}$ of 3 [35]. The linearity was assessed on 10 calibration levels at $0 \mathrm{ng}$ and from 0.1 to $50 \mathrm{ng}$. Absolute recovery rates were determined on labelled compounds (at constant amount of spiking) using matrix and matrixless tests.

\section{Results and discussion}

\subsection{Sample preparation}

\subsubsection{Solvent addition and sensitivity}

PAH quantification requires the addition of native standard solutions in calibration samples and internal standard solution in all the samples. Thus, toluene (solvent used for standard dilutions) 
was added in all samples. This solvent can become a competitor against the target analytes on the adsorbent during the extraction. Then, trapping recoveries of analytes can be affected by the solvent volume added in the sample. In our study, toluene could saturate the adsorbent because of its volatility at 90 or $100^{\circ} \mathrm{C}$ (sample temperatures during extraction). In order to determine the more adapted volume for standard spiking, the first tests were performed without any matrix. Standard solution volumes of 20,50 and $100 \mu \mathrm{L}$ containing the same quantity of PAHs were introduced into headspace vials. We found that the less solvent volume there was, the more intense the signal to noise ratio was for the light PAHs (factor 200 to 7 from naphthalene to pyrene, data not shown). The solvent of the spiking solution, toluene, is also volatile. Thus, there is a competition between toluene and light PAHs, about volatility but above all adsorption on the trap. This explain why, for an equal amount of light PAHs, responses (areas and S/N ratios) increase when the solvent volume decreases. The lowest volume was chosen but we did not try under a $20 \mu \mathrm{L}$ volume to ensure a convenient use and a satisfactory pipetting uncertainty. Therefore, each spiking level, of native and labelled compounds, requires using one spiking solution, in order to ensure that the same volume of $20 \mu \mathrm{L}$ is added. Thus, we prepared ten different solutions: one containing only labelled compounds to spike samples and blanks, and nine solutions containing labelled compounds at the same concentration and native ones at various concentrations.

\subsubsection{Elimination of water traces}

After freeze-drying, the homogenized dried sample was kept at room temperature until the analysis. Dried samples were kept in the freezer for several weeks and samples were slightly rehydrated because of the relative humidity in the closed chamber. Then, traces of moisture led to a plugging, located in the CIS at $\neg 25^{\circ} \mathrm{C}$, by ice formation. To avoid this critical point, we now recommend to perform an additional frozen cycle when dried samples are not analyzed directly after drying. Moreover, we advise to limit the dried samples preservation time after freeze-drying to one week at room temperature to avoid any moisture re-capture. Furthermore, we did not observe any light PAH amounts loss during storage at room temperature. Freeze-drying yields were studied (cf. Fig. S3) by comparing responses of the four lightest labelled PAHs with or without freeze-drying. Results showed that there is no impact on PAHs from 3 cycles, whereas a 2 cycles PAH (NAP) freeze-drying induced some losses. We have to keep this weakness in mind for NAP.

\subsubsection{Sample size optimization}

Several tests with different sample weights $(0.25,0.5,1$ and $2 \mathrm{~g})$ were assessed. Areas of chromatographic peaks were not importantly different between sample sizes (cf. Fig. 2a). However, a higher variability occurred for a sample size of $2 \mathrm{~g}$, probably correlated to the first 
observable matrix effects. Considering the relatively low impact of the sample weight, $1 \mathrm{~g}$ of dried matrix was finally set in the method, to ensure a better representative sample weight and to facilitate the conversion between amount and concentration simultaneously.

\subsection{On-line Dynamic Headspace optimization}

\subsubsection{Nature of the adsorbent}

The nature of the trap adsorbent was assessed on Tenax TA, Tenax GR and Carbopack B/X, after Method 1 extraction on a mussel sample spiked at $20 \mathrm{ng} \mathrm{g}^{-1}$. Tenax TA proved to be an efficient option compared to Tenax GR and Carbopack B/X, particularly for the four lightest PAHs (cf Fig. 2b). Despite a better repeatability of PAH recoveries with the Tenax GR, Tenax TA gave the highest responses, with a gain of $30 \%$ for acenaphtene.

\subsubsection{Incubation parameters}

The incubation allows reaching the equilibrium point of PAH partition between the solid phase (the sample) and the gas phase (headspace). This step remains crucial to lead an efficient headspace extraction afterwards. To reach this equilibrium, we have played on incubation temperature, from 60 to $120^{\circ} \mathrm{C}$ with an incubation time of $10 \mathrm{~min}$ (cf Fig. 2c and $2 \mathrm{~d}$ ), and incubation time, i.e. 10 and $20 \mathrm{~min}$ with an incubation temperature of $100^{\circ} \mathrm{C}$ (cf. Fig. 2e). First of all, Method 1 was applied on a spiked mussel $\left(20 \mathrm{ng} \mathrm{g}^{-1}\right)$ at four incubation temperatures from 90 to $120^{\circ} \mathrm{C}$ by step of $10^{\circ} \mathrm{C}$ (cf. Fig. 2c). The best responses were obtained at $100^{\circ} \mathrm{C}$ for the four lightest PAHs, $90^{\circ} \mathrm{C}$ also being acceptable regarding intensities and standard deviation overlapped with results obtained at $100^{\circ} \mathrm{C}$. The experiment was then reproduced on incurred mussel sample, in order to observe the PAH behaviour in a naturally contaminated matrix (cf. Fig 2d). In this experiment, Method 2 was used. The results confirmed the efficient incubation temperature of $90^{\circ} \mathrm{C}$ for $\mathrm{NAP}, \mathrm{ACY}$ and $\mathrm{ACE}$, while $100^{\circ} \mathrm{C}$ showing that preferable for the heaviest PAHs. Incubation temperatures below $90^{\circ} \mathrm{C}$ were also assessed as they could prevent potential water traces into the headspace, but PAH extraction efficiencies became drastically low from $80^{\circ} \mathrm{C}$, with a loss of $50 \%$ of FLU compared to $100^{\circ} \mathrm{C}$. Therefore, Method 1 was set with an incubation temperature of $100^{\circ} \mathrm{C}$, whereas Method 2, elaborated to minimize water residue, used an incubation temperature of $90^{\circ} \mathrm{C}$. Finally, incubation time above $10 \mathrm{~min}$ was not required as shown in Fig. 2e. This shortest time was chosen to minimize the time of analysis.

\subsubsection{Trapping parameters}

To improve PAH recoveries, we have investigated the parameters to reach an efficient purge of the headspace to move the balance between solid and gas phases, without exceeding the breakthrough volume of the trap. A preliminary experiment was led according to Method 1 without any repetition on a spiked mussel matrix $\left(20 \mathrm{ng} \mathrm{g}^{-1}\right)$ to assess trapping flows between 25 and $75 \mathrm{~mL} \mathrm{~min}^{-1}$ and trapping volumes between 0.25 and $0.75 \mathrm{~L}$ (cf. Fig. 2f). The parameters kept in Method 1 were the last tested, i.e. swept sample headspace by a $0.5 \mathrm{~L}$ of nitrogen at a 
$75 \mathrm{~mL} \mathrm{~min}{ }^{-1}$. As the incubation temperature of Method 1 was set at $100{ }^{\circ} \mathrm{C}$, additional nitrogen was not allowed without capturing water traces from sensitive samples (cf. 3.1.2). However, we investigated using more sweeping gas with Method 2 where the incubation temperature was set at only $90^{\circ} \mathrm{C}$. This time, the experiment was led on an incurred sample $(n=3)$ with trapping flows between 50 and $100 \mathrm{~mL} \mathrm{~min}^{-1}$ and trapping volumes between 0.5 and $2 \mathrm{~L}$ (cf. Fig. $2 \mathrm{~g}$ ). PAH enrichment was proportional to the volume of nitrogen used. We choose to use $2 \mathrm{~L}$ of nitrogen at $100 \mathrm{~mL} \mathrm{m^{-1 }}$ flow to prevent any water traces, without exceeding the breakthrough volume of the trap.

\subsubsection{Dry purge parameters}

Nitrogen volume and flow rate for the dry purge were set according to the Gerstel recommendations in Method $1\left(1 \mathrm{~L}\right.$ at $\left.50 \mathrm{~mL} \mathrm{~min}^{-1}\right)$ [32]. To optimize the drying of the trap, we increased the drying gas volume up to $4 \mathrm{~L}$ at flow rates up to $100 \mathrm{~mL} \mathrm{~min}^{-1}$ (cf. Fig. 2h), without reaching the breakthrough volume. Nevertheless, a $2 \mathrm{~L}$ dry purge volume at a $100 \mathrm{~mL} \mathrm{~min}{ }^{-1}$ dry purge flow rate was chosen to gain time.

\subsubsection{General consideration on DHS optimization}

The DHS optimization was finally a compromise between the highest possible responses for at least the four lightest PAHs without reaching the breakthrough volume, including the lowest moisture content and in the shortest analysis time. Two slightly different combinations of parameters were finally chosen, i.e. Method 1 and Method 2, with complete validation for each one. In summary, Method 2 was chosen to provide a better robustness without the risk of plugging the GC injector with ice, whereas a few samples followed an additional freeze-drying for correct use of the Method 1.

\subsection{Thermo Desorption injection}

TDU and CIS initial temperatures were optimized to improve the chromatographic peak shape, mainly for Naphthalene which is the lightest PAH. Several tests with different initial temperatures of TDU (from 20 to $40^{\circ} \mathrm{C}$ ) and CIS (from $\neg 32^{\circ} \mathrm{C}$ to $\neg 20^{\circ} \mathrm{C}$ ) were assessed (cf. Fig. S4). The lower these temperatures are, the better the shape of the peak is. The optimization gave better results for an initial temperature of the CIS at $\neg 32^{\circ} \mathrm{C}$. However, this temperature was too long to reach for routine purposes. A refocusing temperature of $\neg 25^{\circ} \mathrm{C}$ in the CIS was then chosen (results shown in Fig S1). In parallel, the initial TDU temperature less impacts the chromatographic peak shape than those of the CIS. Then, initial TDU temperature of $30^{\circ} \mathrm{C}$ was set to keep a reasonable cooling delay. Under these conditions, the peak shape of NAP is still improvable. Additional focusing could be achieved by applying a starting oven temperature below $90{ }^{\circ} \mathrm{C}$. However, these lower temperatures were not evaluated in this study. Indeed, this 
peak shape was considered acceptable. In addition, we did not want to lose more time during GC cooling.

Finally, we have optimized the throughput of the analysis. As the bottleneck resulted in the number of traps used, we assessed the intermediate precision using two different traps containing the same adsorbant, i.e. Tenax TA. No significant variability was observed when an incurred mussel sample was analysed with these two different traps (cf. Fig. S5). Therefore, during a sequence, the alternative use of two traps saved time and kept the analysis duration to a minimum, with a total overlap of the next DHS cycle (incubation, trapping and dry purge) with the last GC-MS/MS analysis.

\subsection{MS/MS optimisation}

The mass spectrometry method was developed for light PAHs according to the same principle as described by Veyrand et al. [14] for heaviest ones. Briefly, the acquisition of the signals was ensured in selected reaction monitoring (SRM) following the transitions involving the loss of one $[\mathrm{M}-\mathrm{H}]^{+}$or two hydrogen atoms $\left[\mathrm{M} \neg \mathrm{H}_{2}\right]^{+\bullet}$ from the molecular ion $[\mathrm{M}]^{+\bullet}$, or one or two deuterium atoms for the labeled compounds. Under high energy voltage applied in the collision cell, another specific daughter ion was $\left[\mathrm{M} \neg \mathrm{C}_{2} \mathrm{H}_{2}\right]^{+\bullet}$.

\subsection{Performances}

Results of validations are summarized in Tables 3 and 4. In the light PAHs specific case, the limiting factor was contamination measured by the method blank which represents the environmental contamination level during the batch analysis and which is above $\mathrm{S} / \mathrm{N}$ of 3 . Therefore, LOD were not of interest. LOQ were set at $1 \mathrm{ng}$ in the vial to keep a degree of confidence according to the maximum content of PAH measured in method blanks at $0.67 \mathrm{ng}$. This amount of $1 \mathrm{ng}$ is also the low level of supplemented matrix assays. These limits are fully compatible with the amount range potentially met in seafood [12].

Regarding linearity performances, determination coefficients $\left(\mathrm{R}^{2}\right)$ were better than 0.98 for all analytes, except for phenanthrene in two calibration curves of the second validation.

Absolute recovery rates were determined on labelled compounds (at constant amount of spiking) using matrix and matrixless tests. Indeed, absolute recovery rates in matrix samples on the basis of Dynamic headspace extraction is the ratio of the response of a compound in a matrix test on the response of the same compound in a matrixless test. Results are shown in Table 2. Absolute recovery rates in mussel ground dried samples were between [9-45] \% and [13-62] \% for method Method 1 and method Method 2 respectively. These results were expected since the principle of extraction is based on the balance between the volatility and the adsorption of the compounds in the matrix. Absolute recoveries are classically below 10\% with headspace method, and increase with Dynamic headspace method due to enrichment. In spite of weak values for a few 
compounds, accurate calculated amounts were guaranteed for native compounds by the use of isotope dilution method with labelled compounds corresponding to each native compound. The isotope dilution method allows to automatically correct the amounts in each sample whatever the matrix. Moreover, the GC-MS/MS sensitivity has compensated some low recovery rates to keep satisfactory response levels.The intermediate precision was below $15 \%$ for most of the light PAHs, except for naphthalene, fluorene and phenanthrene in the second validation. The bias values on the supplemented matrix were roughly sufficient, with better results at higher levels of spiking and for Method 1. Quantification in the reference material was within the target range (from 1.5 to $27 \mathrm{ng} \mathrm{g}^{-1}$ ) when given for most light PAHs, except for fluorene in the second validation (cf. Table 4). Indeed, we assume that an unexpected contamination could occur in the sample without any detection in the blank sample. The result is then an extreme bias value. Taking into account all uncertainties contributions, including the uncertainty component associated to the concentrations of the standard solutions (3.2/3.2/3.3\% at low/middle/high level), the expanded measurement uncertainties, with a coverage factor of 2 (confidence level of 95\%), were ranging from 8 to $73 \%$ for Method 1, depending on the quantified analytes and the level. Method 2 gave higher uncertainties, particularly for fluorene. We assume that a kind of unexpected contamination occurs frequently for PHE and FLU. Indeed, their intermediate precision error and associated bias were relatively high, leading to extremely high-expanded uncertainties, particularly when method 2 is used. The next stage is now to monitor this suspected contamination to treat its sources. For the next batches of analysis, a recommendation could be to use Method 2 in routine analysis to prevent any ice formation in the CIS and to use Method 1 for confirmatory purposes, particularly for fluorene quantification, in order to increase the intermediate precision of measurement.

\subsection{Light PAHs Occurrence}

Analyses were performed with Method 1 on almost two hundred samples of molluscs, echinoderms and fishes. The aim of this work was to assess the methodology and to estimate the order of magnitude of light PAHs amounts in seafood simultaneously. The four lightest PAHs amounts were reported in Fig. 3, taking a value equal to zero when the compound was not quantified (lowerbound). These results have highlighted a quantification frequency greater than $66 \%$ for naphthalene and fluorene at amounts of up to $8 \mathrm{ng} \mathrm{g}^{-1}$ of dry matter.

\section{Conclusions}

The work carried out has led to develop two methods to determine light PAHs both in the mollusc matrix and in other matrices such as oils, then proceeding by standard addition method. The developed methods were validated for the lightest PAHs: NAP, ACY, ACE, FLU but also PHE and ANT. Analytical performances matched with the levels of contamination observed in 
seafood. In addition, the first analyses produced with this method show the interest of continuing to monitor these parameters in seafood and more generally on a wide range of foodstuffs in order to better characterize the associated risk to light PAHs dietary exposure.

\section{Acknowledgements}

We thank the technical staff of Research Institute for Chromatography (Saint-Priest, France) for their help and wise advice, and the French Ministry of Agriculture for its financial support. 


\section{References}

[1] X.-C. Wang, Y.-X. Zhang, F.R. Chen, Distribution and partitioning of polycyclic aromatic hydrocarbons (PAHs) in Different Size Fractions in Sediments from Boston Harbor, United States, Mar. Pollut. Bull., 42 (2001) 1139-1149.

[2] R. Gioia, E. Steinnes, G.O. Thomas, S.N. Mejier, K.C. Jones, Persistent organic pollutants in European background air: derivation of temporal and latitudinal trends, J. Environ. Monit., 8 (2006) 700-710.

[3] L. Han, J. Bai, Z. Gao, W. Wang, D. Wang, B. Cui, X. Liu, Polycyclic aromatic hydrocarbons (PAHs) in surface soils from reclaimed and ditch wetlands along a 100-year chronosequence of reclamation in a Chinese estuary: Occurrence, sources, and risk assessment, Agric., Ecosyst. Environ., 286 (2019) 106648.

[4] V. Varlet, T. Serot, F. Monteau, B. Le Bizec, C. Prost, Determination of PAH profiles by GC-MS/MS in salmon processed by four cold-smoking techniques, Food Addit. Contam., 24 (2007) 744-757.

[5] P.E. Rosenfeld, L.G.H. Feng, 15 - Bioaccumulation of Dioxins, PCBs, and PAHs, in: P.E. Rosenfeld, L.G.H. Feng (Eds.) Risks of Hazardous Wastes, William Andrew Publishing, Boston, 2011, pp. 201-213.

[6] C.E. Bostrom, P. Gerde, A. Hanberg, B. Jernstrom, C. Johansson, T. Kyrklund, A. Rannug, M. Tornqvist, K. Victorin, R. Westerholm, Cancer risk assessment, indicators, and guidelines for polycyclic aromatic hydrocarbons in the ambient air, Environ. Health Perspect., 110 Suppl 3 (2002) 451-488.

[7] E. Drwal, A. Rak, E.L. Gregoraszczuk, Review: Polycyclic aromatic hydrocarbons (PAHs)Action on placental function and health risks in future life of newborns, Toxicology, 411 (2019) 133-142.

[8] K.H. Kim, S.A. Jahan, E. Kabir, R.J. Brown, A review of airborne polycyclic aromatic hydrocarbons (PAHs) and their human health effects, Environ. Int., 60 (2013) 71-80.

[9] B. Veyrand, V. Sirot, S. Durand, C. Pollono, P. Marchand, G. Dervilly-Pinel, A. Tard, J.C. Leblanc, B. Le Bizec, Human dietary exposure to polycyclic aromatic hydrocarbons: results of the second French Total Diet Study, Environ. Int., 54 (2013) 11-17.

[10] IARC, Agents Classified by the IARC Monographs, Volumes 1-123, in, 2018.

[11] Y. Zhang, J. Ding, G. Shen, J. Zhong, C. Wang, S. Wei, C. Chen, Y. Chen, Y. Lu, H. Shen, W. Li, Y. Huang, H. Chen, S. Su, N. Lin, X. Wang, W. Liu, S. Tao, Dietary and inhalation exposure to polycyclic aromatic hydrocarbons and urinary excretion of monohydroxy metabolites - A controlled case study in Beijing, China, Environ. Pollut., 184 (2014) 515-522.

[12] R. Marti-Cid, J.M. Llobet, V. Castell, J.L. Domingo, Evolution of the dietary exposure to polycyclic aromatic hydrocarbons in Catalonia, Spain, Food Chem. Toxicol., 46 (2008) 31633171.

[13] P.K. Thai, A.L. Heffernan, L.L. Toms, Z. Li, A.M. Calafat, P. Hobson, S. Broomhall, J.F. Mueller, Monitoring exposure to polycyclic aromatic hydrocarbons in an Australian population using pooled urine samples, Environ. Int., 88 (2016) 30-35.

[14] B. Veyrand, A. Brosseaud, L. Sarcher, V. Varlet, F. Monteau, P. Marchand, F. Andre, B. Le Bizec, Innovative method for determination of 19 polycyclic aromatic hydrocarbons in food and oil samples using gas chromatography coupled to tandem mass spectrometry based on an isotope dilution approach, J. Chromatogr. A, 1149 (2007) 333-344.

[15] P. Plaza-Bolanos, A.G. Frenich, J.L. Vidal, Polycyclic aromatic hydrocarbons in food and beverages. Analytical methods and trends, J. Chromatogr. A, 1217 (2010) 6303-6326.

[16] V. Andreu, Y. Picó, Pressurized liquid extraction of organic contaminants in environmental and food samples, TrAC, Trends Anal. Chem., 118 (2019) 709-721. 
[17] M. Masuda, Q. Wang, M. Tokumura, Y. Miyake, T. Amagai, Simultaneous determination of polycyclic aromatic hydrocarbons and their chlorinated derivatives in grilled foods, Ecotoxicol. Environ. Saf., 178 (2019) 188-194.

[18] G. Purcaro, S. Moret, L.S. Conte, Overview on polycyclic aromatic hydrocarbons: occurrence, legislation and innovative determination in foods, Talanta, 105 (2013) 292-305.

[19] S. Vichi, L. Pizzale, L.S. Conte, S. Buxaderas, E. Lopez-Tamames, Simultaneous determination of volatile and semi-volatile aromatic hydrocarbons in virgin olive oil by headspace solid-phase microextraction coupled to gas chromatography/mass spectrometry, J. Chromatogr. A, 1090 (2005) 146-154.

[20] N. Aguinaga, N. Campillo, P. Vinas, M. Hernandez-Cordoba, Determination of 16 polycyclic aromatic hydrocarbons in milk and related products using solid-phase microextraction coupled to gas chromatography-mass spectrometry, Anal. Chim. Acta, 596 (2007) 285-290.

[21] N. Aguinaga, N. Campillo, P. Vinas, M. Hernandez-Cordoba, Evaluation of solid-phase microextraction conditions for the determination of polycyclic aromatic hydrocarbons in aquatic species using gas chromatography, Anal. Bioanal. Chem., 391 (2008) 1419-1424.

[22] G. Purcaro, P. Morrison, S. Moret, L.S. Conte, P.J. Marriott, Determination of polycyclic aromatic hydrocarbons in vegetable oils using solid-phase microextraction-comprehensive twodimensional gas chromatography coupled with time-of-flight mass spectrometry, J. Chromatogr. A, 1161 (2007) 284-291.

[23] D. Martin, J. Ruiz, Analysis of polycyclic aromatic hydrocarbons in solid matrixes by solidphase microextraction coupled to a direct extraction device, Talanta, 71 (2007) 751-757.

[24] M.R. Afshar Mogaddam, A. Mohebbi, A. Pazhohan, F. Khodadadeian, M.A. Farajzadeh, Headspace mode of liquid phase microextraction: A review, TrAC, Trends Anal. Chem., 110 (2019) 8-14.

[25] A.A. Rincon, V. Pino, J.H. Ayala, A.M. Afonso, Headspace-single drop microextraction (HS-SDME) in combination with high-performance liquid chromatography (HPLC) to evaluate the content of alkyl- and methoxy-phenolic compounds in biomass smoke, Talanta, 85 (2011) $1265-1273$.

[26] B. Gaspardo, G. Procida, B. Toso, B. Stefanon, Determination of volatile compounds in San Daniele ham using headspace GC-MS, Meat Sci, 80 (2008) 204-209.

[27] R. Barcarolo, P. Casson, Modified Capillary GCMS System Enabling Dynamic Headspace Sampling with On-Line Cryofocusing and Cold On-Column Injection of Liquid Samples, J. High. Resolut. Chromatogr., 20 (1997) 24-28.

[28] A. Marquez, M.P. Serratosa, J. Merida, L. Zea, L. Moyano, Optimization and validation of an automated DHS-TD-GC-MS method for the determination of aromatic esters in sweet wines, Talanta, 123 (2014) 32-38.

[29] L. Moyano, M.P. Serratosa, A. Marquez, L. Zea, Optimization and validation of a DHS-TDGC-MS method to wineomics studies, Talanta, 192 (2019) 301-307.

[30] C. Sales, T. Portoles, L.G. Johnsen, M. Danielsen, J. Beltran, Olive oil quality classification and measurement of its organoleptic attributes by untargeted GC-MS and multivariate statisticalbased approach, Food Chem., 271 (2019) 488-496.

[31] A. Erb, P. Marsan, M. Burgart, A. Remy, A.M. Lambert-Xolin, F. Jeandel, O. Hanser, A. Robert, Simultaneous determination of aromatic and chlorinated compounds in urine of exposed workers by dynamic headspace and gas chromatography coupled to mass spectrometry (dHSGC-MS), J Chromatogr B Analyt Technol Biomed Life Sci, 1125 (2019) 121724.

[32] J. Tsunokawa, N. Ochiai, K. Sasamoto, A. Hoffmann, 2-Step Multi-Volatile Method (2-Step MVM) for Characterization of Aroma Compounds in Bread, in: Gerstel Application Note, 2016. [33] J.R. Stuff, J.A. Whitecavage, E.A. Pfannkoch, Off-gassing of Rubber Particles Used for Athletic Fields using Automated Dynamic Headspace Sampling, in: Gerstel Application Note, 2016. 
[34] E. Communities, Commission decision of 12 August 2002 implementing Council Directive 96/23/EC concerning the performance of analytical methods and the interpretation of results, in: O.J.o.t.E. Communities (Ed.) 2002/657/EC, 2002.

[35] 2017/771, Commission Regulation (EU) 2017/771 amending Regulation (EC) No 152/2009 as regards the methods for the determination of the levels of dioxins and polychlorinated biphenyls, 2017 O.J. L 115/22 (Text with EEA relevance), in, 2017. 


\section{Figure Captions}<smiles>c1ccc2ccccc2c1</smiles>

Naphthalene (NAP)<smiles>C1=Cc2cccc3cccc1c23</smiles>

Acenaphthylene (ACY)

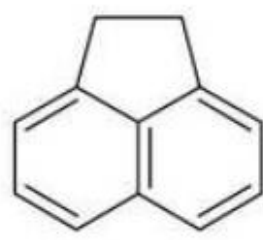

Acenaphthene

(ACE)
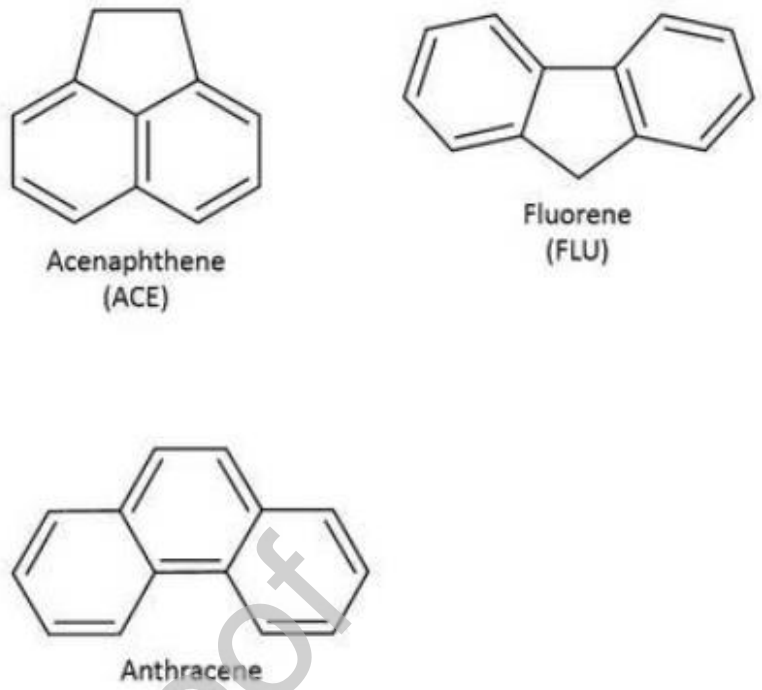

(ANT)<smiles>c1ccc2cc3ccccc3cc2c1</smiles>

(ANT)

Fig. 1. Chemical structure of the light PAHs

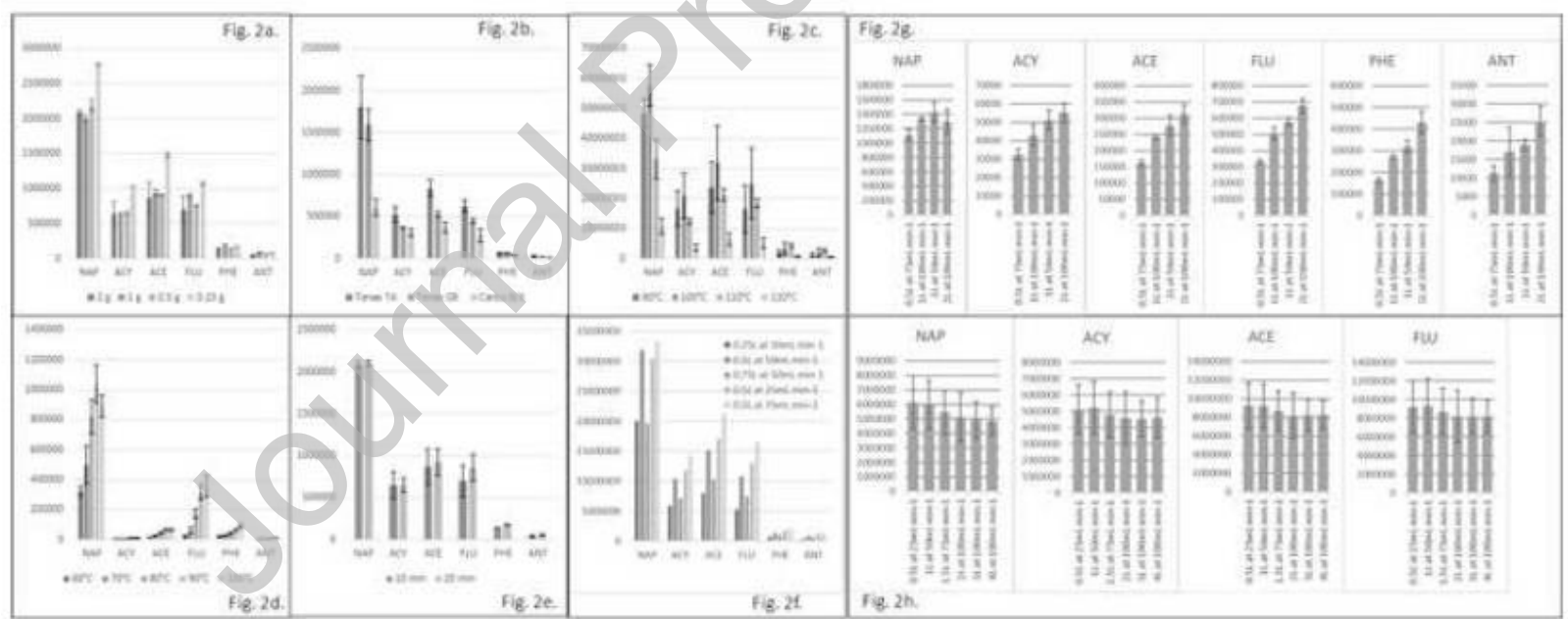

Fig. 2. (a) PAHs response in matrix spiked at $1 \mathrm{ng}$ regardless of the sample weight $(\mathrm{n}=2$; SD in brackets); (b) PAHs response in matrix spiked at $20 \mathrm{ng}$ in relation to adsorbent types $(\mathrm{n}=3$; SD in brackets); PAHs response (c) in matrix spiked at $20 \mathrm{ng}$ and (d) in incurred mussel in relation to incubation temperature ( $\mathrm{n}=2(\mathrm{c})$ and $\mathrm{n}=3(\mathrm{~d})$; SD in brackets); (e) PAHs response in matrix spiked at $20 \mathrm{ng}$ in relation to incubation times ( $\mathrm{n}=2$; SD in brackets); (f) PAHs response in matrix spiked at $20 \mathrm{ng}$ in relation to traping volume and flow rates; (g) PAHs response in incurred mussel sample in relation to trapping volume and trapping flow $(\mathrm{n}=3 ; \mathrm{SD}$ in brackets); (h) PAHs response in matrix spiked at $2 \mathrm{ng}$ in relation to drying purge volume and flow rate $(\mathrm{n}=$ 3 ; SD in brackets) 


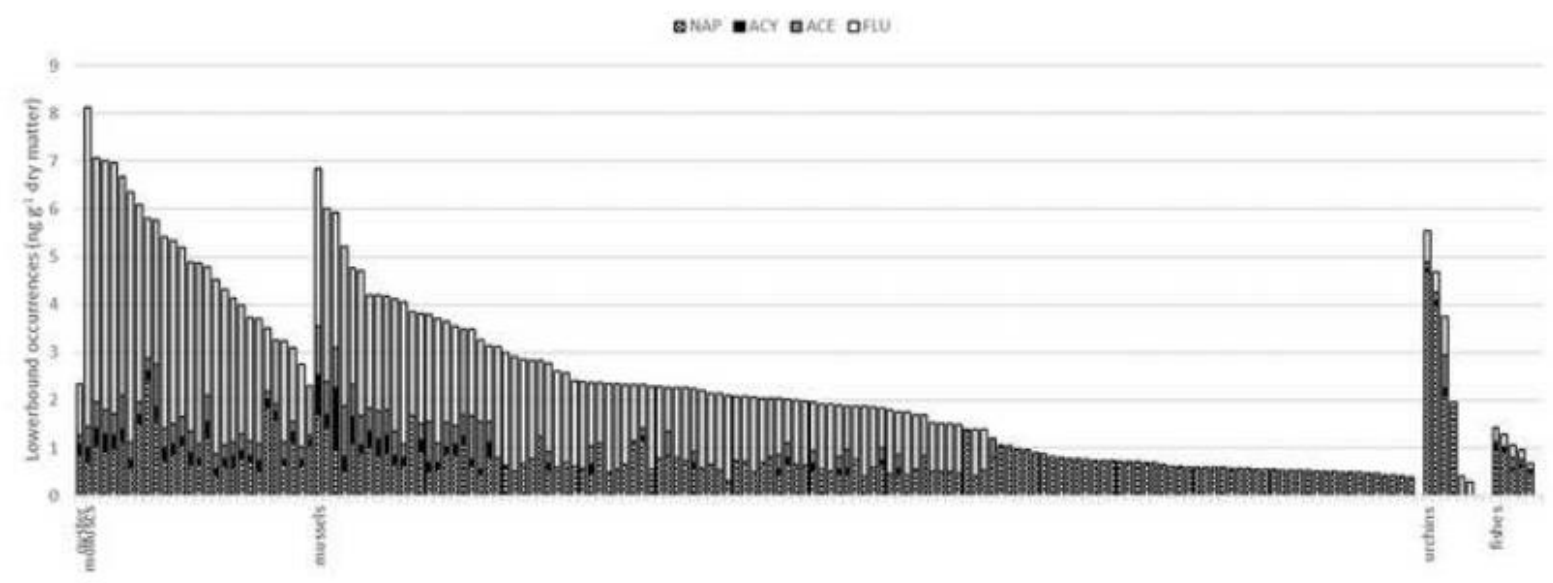

Fig. 3. Lowerbound occurrences in $\mathrm{ng} \mathrm{g}^{-1}$ of dry matter by matrix type

\section{Table list}

Table 1: Monitored transitions, indicative ratios and retention times of light PAHs. (IS : Internal Standard ; A : Analyte ; RT : retention time.)

Table 2 : Absolute recovery rates of labelled light PAHs (determined at constant amount using matrix and matrixless tests).

Table 3: Performances of light PAHs for methods 1 and 2. Uncertainties are given for each level of concentration and average uncertainty is indicated in bold (Low level (1 ng)/Middle level (5 ng)/High level (20 ng)/Average)

Table 4: quantified values vs. reference values in RM IAEA-432. $(\checkmark$ : in the target range; $\times$ : out the target range.)

Credit Author statement

Ingrid GUIFFARD : Writing - Original Draft, Conceptualization, Methodology, Validation, Formal analysis, Investigation, Visualization

Thomas GENY ; Formal analysis

Bruno VEYRAND : Formal analysis

Philippe MARCHAND : Resources

Anne PELLOUIN-GROUHEL : Resources

Bruno LE BIZEC : Resources

Emmanuelle BICHON : Writing - Review \& Editing, Conceptualization, Methodology, Validation, Investigation, Supervision

Table 1: Monitored transitions, indicative ratios and retention times of light PAHs. (IS : Internal Standard ; A : Analyte ; RT : retention time.)

\begin{tabular}{|c|c|c|c|c|c|c|c|c|}
\hline Compounds & Type & Transition 1 & $\begin{array}{c}\text { Collision } \\
\text { T1 (eV) }\end{array}$ & Transition 2 & $\begin{array}{c}\text { Collision } \\
\text { T2 (eV) }\end{array}$ & $\begin{array}{c}\text { Indicative } \\
\text { Ratio } \\
\text { (T2/T1) }\end{array}$ & $\begin{array}{l}\text { Indicative } \\
\text { RT (min) }\end{array}$ & Window \\
\hline Naphthalene-d 8 & IS & $136 . .1>108 . .1$ & 25 & $136 . .1>134 . .1$ & 20 & & $6 . .40$ & 1 \\
\hline Naphthalene & A & $128 . .1>102 . .0$ & 25 & $128 . .1>127 . .1$ & 20 & 0.473 & $6 . .43$ & \\
\hline
\end{tabular}




\begin{tabular}{lccccccc} 
Acenaphthylene- $\mathrm{d}_{8}$ & IS & $160 . .1>158 . .1$ & 25 & $160 . .1>132 . .1$ & 30 & $9 . .83$ & $\mathbf{2}$ \\
Acenaphthylene & A & $152 . .1>151 . .1$ & 25 & $152 . .1>126 . .0$ & 30 & 0.439 & $9 . .86$ \\
\hline Acenaphthene-d $\mathrm{d}_{10}$ & IS & $164 . .1>162 . .1$ & 20 & $164 . .1>160 . .1$ & 40 & $10 . .17$ & $\mathbf{3}$ \\
Acenaphthene & A & $154 . .1>153 . .1$ & 20 & $154 . .1>152 . .1$ & 40 & 0.553 & $10 . .24$ \\
\hline Fluorene- $\mathrm{d}_{10}$ & IS & $176 . .1>174 . .1$ & 25 & $176 . .1>172 . .1$ & 40 & $11 . .42$ & $\mathbf{4}$ \\
Fluorene & A & $166 . .1>165 . .1$ & 25 & $166 . .1>164 . .1$ & 40 & 0.189 & $11 . .48$ \\
\hline Phenanthrene- $\mathrm{d}_{10}$ & IS & $188 . .1>160 . .1$ & 30 & $188 . .1>184 . .1$ & 35 & $13 . .72$ & $\mathbf{5}$ \\
Phenanthrene & A & $178 . .1>152 . .1$ & 30 & $178 . .1>176 . .1$ & 35 & 1.106 & $13 . .76$ \\
Anthracene- $\mathrm{d}_{10}$ & IS & $188 . .1>160 . .1$ & 30 & $188 . .1>184 . .1$ & 35 & & $13 . .86$ \\
Anthracene & A & $178 . .1>152 . .1$ & 30 & $178 . .1>176 . .1$ & 35 & 1.305 & $13 . .91$ \\
\hline
\end{tabular}

Table 2 : Absolute recovery rates of labelled light PAHs (determined at constant amount using matrix and matrixless tests).

\begin{tabular}{lcc}
\hline \multicolumn{1}{c}{ Compounds } & \multicolumn{1}{c}{$\begin{array}{c}\text { Absolute recovery rate } \\
\text { with Method 1 parameters validation }\end{array}$} & $\begin{array}{c}\text { Absolute recovery rate } \\
\text { with Method 2 parameters validation }\end{array}$ \\
\hline Naphthalene- $\mathrm{d}_{8}$ & $45 \%$ & $13 \%$ \\
Acenaphthylene- $\mathrm{d}_{8}$ & $30 \%$ & $62 \%$ \\
Acenaphthene- $\mathrm{d}_{10}$ & $37 \%$ & $53 \%$ \\
Fluorene- $\mathrm{d}_{10}$ & $25 \%$ & $41 \%$ \\
Phenanthrene- $\mathrm{d}_{10}$ & $9 \%$ & $21 \%$ \\
Anthracene- $\mathrm{d}_{10}$ & $11 \%$ & $36 \%$ \\
\hline
\end{tabular}


Table 3: Performances of light PAHs for Methods 1 and 2. Uncertainties are given for each level of concentration and average uncertainty is indicated in bold (Low level (1 ng)/Middle level (5 ng)/High level (20 ng)/Average)

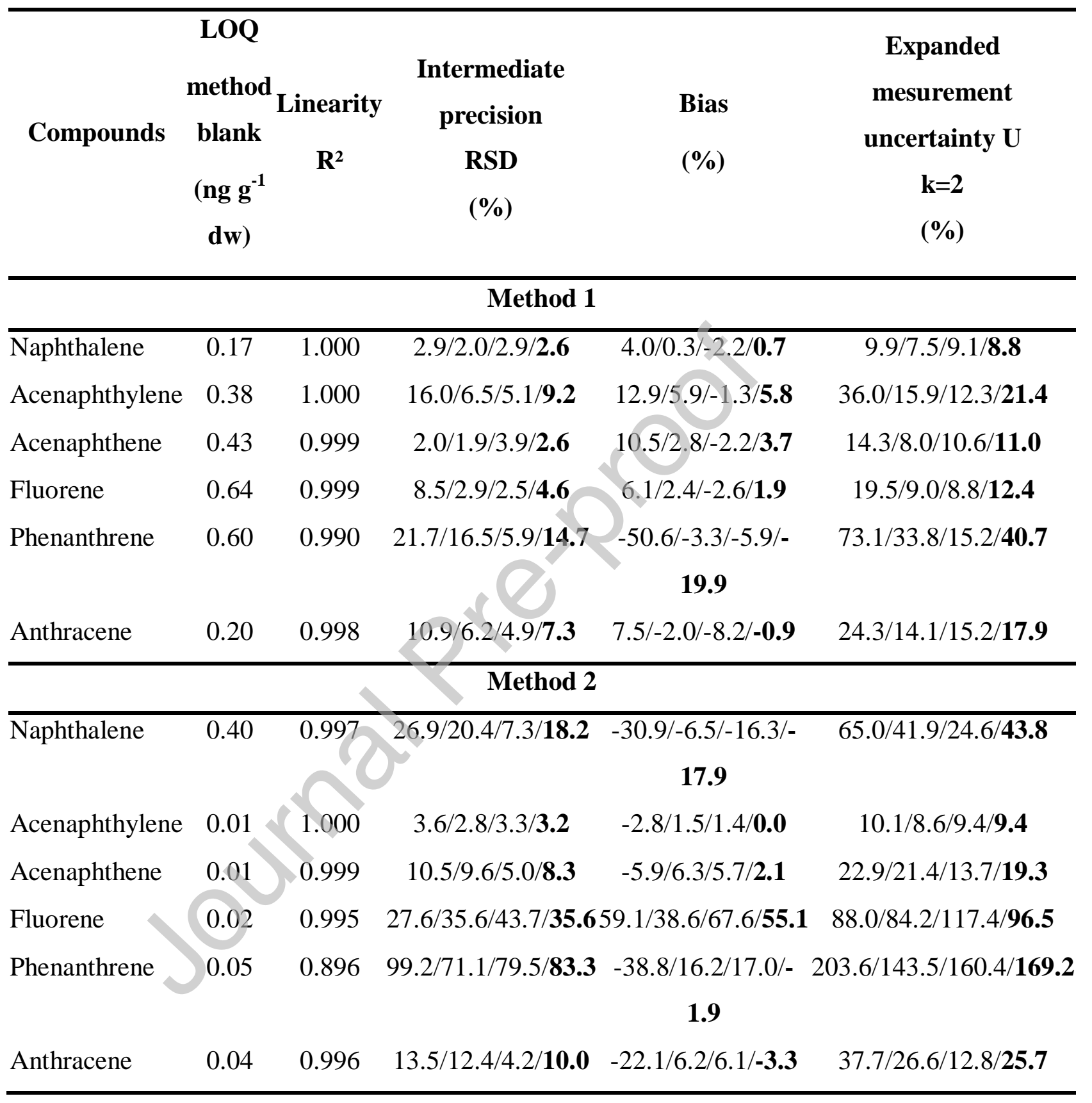


Table 4: quantified values vs. reference values in RM IAEA-432. $(\checkmark$ : in the target range ; $\times$ : out the target range.)

\begin{tabular}{lccccc}
\hline Compounds & $\begin{array}{c}\text { Reference } \\
\text { values } \\
\left(\mathbf{n g ~ g}^{-\mathbf{1}}\right)\end{array}$ & $\begin{array}{c}\text { Amounts } \\
\text { with Method 1 } \\
\text { parameters } \\
\text { validation } \\
\left(\mathbf{n g ~ g}^{-1}\right)\end{array}$ & $\begin{array}{c}\text { Amounts } \\
\text { with Method 2 } \\
\text { parameters } \\
\text { validation } \\
\left(\mathbf{n g ~ g}^{-1}\right)\end{array}$ & $\begin{array}{c}\text { Bias } \\
\text { with Method 1 } \\
\text { parameters } \\
\text { validation }\end{array}$ & $\begin{array}{c}\text { Bias } \\
\text { with Method 2 } \\
\text { parameters } \\
\text { validation }\end{array}$ \\
\hline Naphthalene & $15 \pm 18$ & $13 \checkmark$ & $7 \checkmark$ & -11 & $(\boldsymbol{\%})$ \\
Acenaphthylene & - & 1.8 & 0.3 & - & -53 \\
Acenaphthene & - & 2.5 & 0.7 & - & - \\
Fluorene & $4.1 \pm 2.2$ & $3.9 \checkmark$ & $26.2 \times$ & -5 & - \\
Phenanthrene & $27 \pm 21$ & $16 \checkmark$ & $8 \checkmark$ & -39 & -69 \\
Anthracene & $1.5 \pm 1.1$ & $1.1 \checkmark$ & $1.0 \checkmark$ & -25 & -36 \\
\hline
\end{tabular}

